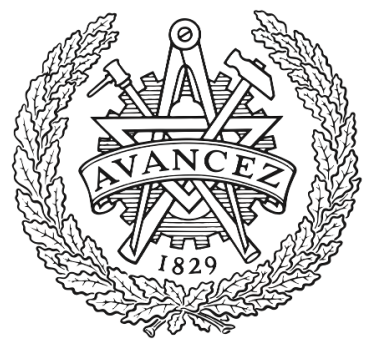

CHALMERS

UNIVERSITY OF TECHNOLOGY

\title{
K2-155: A Bright Metal-poor M Dwarf with Three Transiting Super-Earths
}

Downloaded from: https://research.chalmers.se, 2023-04-26 13:52 UTC

Citation for the original published paper (version of record):

Hirano, T., Dai, F., Livingston, J. et al (2018). K2-155: A Bright Metal-poor M Dwarf with Three

Transiting Super-Earths. Astronomical Journal, 155(3). http://dx.doi.org/10.3847/1538-3881/aaaa6e

N.B. When citing this work, cite the original published paper. 


\title{
K2-155: A Bright Metal-poor M Dwarf with Three Transiting Super-Earths
}

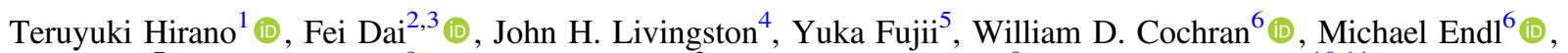 \\ Davide Gandolfi ${ }^{7}$ (10), Seth Redfield ${ }^{8}$ (10), Joshua N. Winn ${ }^{3}$ (1) , Eike W. Guenther ${ }^{9}$, Jorge Prieto-Arranz ${ }^{10,11}$, Simon Albrecht ${ }^{12}$, \\ Oscar Barragan $^{7}$, Juan Cabrera ${ }^{13}$ (D), P. Wilson Cauley ${ }^{14}$, Szilard Csizmadia ${ }^{13}$, Hans Deeg ${ }^{10,11}$ (D), Philipp Eigmüller ${ }^{13}$,

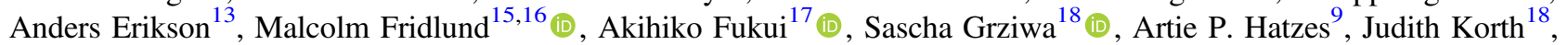 \\ Norio Narita $^{4,19,20}$ (1) , David Nespral ${ }^{10,11}$, Prajwal Niraula ${ }^{8}$, Grzegorz Nowak ${ }^{10,11}$ (i) , Martin Pätzold ${ }^{18}$, Enric Palle , $^{10,11}$, \\ Carina M. Persson ${ }^{16}$, Heike Rauer ${ }^{13,21}$, Ignasi Ribas ${ }^{22}$ (1), Alexis M. S. Smith ${ }^{13}$, and Vincent Van Eylen ${ }^{15}$ \\ ${ }^{1}$ Department of Earth and Planetary Sciences, Tokyo Institute of Technology, 2-12-1 Ookayama, Meguro-ku, Tokyo 152-8551, Japan; hirano@geo.titech.ac.jp \\ ${ }_{2}^{2}$ Department of Physics, and Kavli Institute for Astrophysics and Space Research, Massachusetts Institute of Technology, Cambridge, MA 02139, USA \\ ${ }^{3}$ Department of Astrophysical Sciences, Princeton University, 4 Ivy Lane, Princeton, NJ 08544, USA \\ ${ }^{4}$ Department of Astronomy, Graduate School of Science, The University of Tokyo, Hongo 7-3-1, Bunkyo-ku, Tokyo, 113-0033, Japan \\ ${ }^{5}$ Earth-Life Science Institute, Tokyo Institute of Technology, Tokyo, 152-8550, Japan \\ ${ }^{6}$ Department of Astronomy and McDonald Observatory, University of Texas at Austin, 2515 Speedway, Stop C1400, Austin, TX 78712, USA \\ ${ }^{7}$ Dipartimento di Fisica, Università di Torino, via P. Giuria 1, I-10125 Torino, Italy \\ ${ }^{8}$ Astronomy Department and Van Vleck Observatory, Wesleyan University, Middletown, CT 06459, USA \\ 9 Thüringer Landessternwarte Tautenburg, Sternwarte 5, D-07778 Tautenberg, Germany \\ ${ }_{11}^{10}$ Instituto de Astrofísica de Canarias, C/ Vía Láctea s/n, E-38205 La Laguna, Spain \\ ${ }^{11}$ Departamento de Astrofísica, Universidad de La Laguna, E-38206 La Laguna, Spain \\ ${ }^{12}$ Stellar Astrophysics Centre, Department of Physics and Astronomy, Aarhus University, Ny Munkegade 120, DK-8000 Aarhus C, Denmark \\ ${ }^{13}$ Institute of Planetary Research, German Aerospace Center, Rutherfordstrasse 2, D-12489 Berlin, Germany \\ ${ }^{14}$ School of Earth and Space Exploration, Arizona State University, Tempe, AZ 85281, USA \\ ${ }^{15}$ Leiden Observatory, Leiden University, 2333CA Leiden, The Netherlands \\ ${ }^{16}$ Department of Space, Earth and Environment, Chalmers University of Technology, Onsala Space Observatory, SE-439 92 Onsala, Sweden \\ ${ }^{17}$ Okayama Astrophysical Observatory, National Astronomical Observatory of Japan, Asakuchi, Okayama 719-0232, Japan \\ ${ }^{18}$ Rheinisches Institut für Umweltforschung an der Universität zu Köln, Aachener Strasse 209, D-50931 Köln, Germany \\ ${ }^{19}$ Astrobiology Center, NINS, 2-21-1 Osawa, Mitaka, Tokyo 181-8588, Japan \\ ${ }^{20}$ National Astronomical Observatory of Japan, NINS, 2-21-1 Osawa, Mitaka, Tokyo 181-8588, Japan \\ ${ }^{21}$ Center for Astronomy and Astrophysics, TU Berlin, Hardenbergstr. 36, D-10623 Berlin, Germany \\ ${ }^{22}$ Institut de Ciències de l'Espai (CSIC-IEEC), Carrer de Can Magrans, Campus UAB, E-08193 Bellaterra, Spain \\ Received 2017 November 9; revised 2018 January 21; accepted 2018 January 22; published 2018 February 23
}

\begin{abstract}
We report on the discovery of three transiting super-Earths around K2-155 (EPIC 210897587), a relatively bright early M dwarf ( $V=12.81 \mathrm{mag}$ ) observed during Campaign 13 of the NASA $K 2 \mathrm{mission}$. To characterize the system and validate the planet candidates, we conducted speckle imaging and high-dispersion optical spectroscopy, including radial velocity measurements. Based on the $K 2$ light curve and the spectroscopic characterization of the host star, the planet sizes and orbital periods are $1.55_{-0.17}^{+0.20} R_{\oplus}$ and $6.34365 \pm 0.00028$ days for the inner planet; $1.95_{-0.22}^{+0.27} R_{\oplus}$ and $13.85402 \pm 0.00088$ days for the middle planet; and $1.64_{-0.17}^{+0.18} R_{\oplus}$ and $40.6835 \pm 0.0031$ days for the outer planet. The outer planet (K2-155d) is near the habitable zone, with an insolation $1.67 \pm 0.38$ times that of the Earth. The planet's radius falls within the range between that of smaller rocky planets and larger gas-rich planets. To assess the habitability of this planet, we present a series of three-dimensional global climate simulations, assuming that K2-155d is tidally locked and has an Earth-like composition and atmosphere. We find that the planet can maintain a moderate surface temperature if the insolation proves to be smaller than $\sim 1.5$ times that of the Earth. Doppler mass measurements, transit spectroscopy, and other follow-up observations should be rewarding, as K2-155 is one of the optically brightest $\mathrm{M}$ dwarfs known to harbor transiting planets.
\end{abstract}

Key words: planets and satellites: detection - stars: individual (K2-155 = EPIC 210897587) - techniques: photometric - techniques: radial velocities - techniques: spectroscopic

\section{Introduction}

Nearby stars are always attractive targets for the characterization of exoplanets of all sizes. Nearby $M$ dwarfs are especially attractive because their small sizes lead to larger transit and Doppler signals, and because the habitable zone occurs at relatively short orbital periods. However, the number of optically bright $\mathrm{M}$ dwarfs known to have transiting planets is still small. As of 2018 January, there are only a handful of transiting planets orbiting $\mathrm{M}$ dwarfs that are bright enough for further follow-up observations (e.g., $V \lesssim 14$ mag; Butler et al. 2004; Bonfils et al. 2012; Berta-Thompson et al. 2015; Crossfield et al. 2015).
After the failure of two reaction wheels, the Kepler spacecraft ended its original mission and was repurposed to conduct another transit survey known as the $K 2$ mission (Howell et al. 2014). This new survey is examining a series of star fields around the ecliptic. Together these fields cover a much wider area of the sky than the original mission, but each field is observed for a shorter duration (about 80 days) than the original mission (4 years). Because of the wider sky coverage, it has been possible to observe a larger sample of bright and nearby stars. This has led to many new planet discoveries, including planets around low-mass stars (see, e.g., Crossfield et al. 2015; Montet et al. 2015; Hirano et al. 2016; Dressing et al. 2017). 
Table 1

Stellar Parameters of K2-155

\begin{tabular}{|c|c|c|}
\hline Parameter & Value & Source \\
\hline \multicolumn{3}{|l|}{ (Identifiers) } \\
\hline EPIC & 210897587 & \\
\hline 2MASS & $\mathrm{J} 04215245+2121131$ & \\
\hline \multicolumn{3}{|c|}{ (Stellar Parameters from the Literature) } \\
\hline R.A. (J2000) & $04: 21: 52.49$ & UCAC5 \\
\hline Decl. (J2000) & $+21: 21: 12.95$ & UCAC5 \\
\hline$\mu_{\alpha} \cos \delta\left(\operatorname{mas} \mathrm{yr}^{-1}\right)$ & $199.6 \pm 1.3$ & UCAC5 \\
\hline 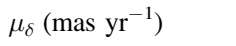 & $-77.3 \pm 1.3$ & UCAC5 \\
\hline$B(\operatorname{mag})$ & $14.073 \pm 0.051$ & APASS \\
\hline$V(\mathrm{mag})$ & $12.806 \pm 0.046$ & APASS \\
\hline$g^{\prime}(\mathrm{mag})$ & $13.491 \pm 0.047$ & APASS \\
\hline$r^{\prime}(\mathrm{mag})$ & $12.286 \pm 0.059$ & APASS \\
\hline$J$ (mag) & $10.274 \pm 0.024$ & 2MASS \\
\hline$H$ (mag) & $9.686 \pm 0.022$ & 2MASS \\
\hline$K_{\mathrm{s}}(\mathrm{mag})$ & $9.496 \pm 0.017$ & 2MASS \\
\hline$W 1$ (mag) & $9.435 \pm 0.023$ & WISE \\
\hline$W 2$ (mag) & $9.404 \pm 0.019$ & $W I S E$ \\
\hline W3 (mag) & $9.343 \pm 0.041$ & WISE \\
\hline W4 (mag) & $>8.317$ & $W I S E$ \\
\hline
\end{tabular}

(Spectroscopic and Derived Parameters)

\begin{tabular}{lcr}
$T_{\text {eff }}(\mathrm{K})$ & $3919 \pm 70$ & This work \\
{$[\mathrm{Fe} / \mathrm{H}](\mathrm{dex})$} & $-0.42 \pm 0.12$ & This work \\
$R_{\star}\left(R_{\odot}\right)$ & $0.526 \pm 0.053$ & This work \\
$M_{\star}\left(M_{\odot}\right)$ & $0.540 \pm 0.056$ & This work \\
$\log g(\mathrm{cgs})$ & $4.732 \pm 0.046$ & This work \\
$\rho_{\star}\left(\rho_{\odot}\right)$ & $3.84 \pm 0.79$ & This work \\
$L_{\star}\left(L_{\odot}\right)$ & $0.059 \pm 0.013$ & This work \\
$\operatorname{distance~}(\mathrm{pc})$ & $62.3 \pm 9.3$ & This work \\
$\mathrm{RV}\left(\mathrm{km} \mathrm{s}^{-1}\right)$ & $19.34 \pm 0.16$ & This work \\
$U\left(\mathrm{~km} \mathrm{~s}^{-1}\right)$ & $24.6 \pm 2.2$ & This work \\
$V\left(\mathrm{~km} \mathrm{~s}^{-1}\right)$ & $-39.9 \pm 8.2$ & This work \\
$W\left(\mathrm{~km} \mathrm{~s}^{-1}\right)$ & $27.5 \pm 4.1$ & This work \\
\hline
\end{tabular}

KESPRINT is one of several large collaborations that are detecting planet candidates using $K 2$ data and performing follow-up observations to validate the candidates and measure planet masses (see, e.g., Fridlund et al. 2017; Gandolfi et al. 2017; Guenther et al. 2017; Livingston et al. 2017). This latest KESPRINT paper focuses on K2-155 (EPIC 210897587), a bright $\mathrm{M}$ dwarf $(V=12.81)$ observed during Campaign 13 of the $K 2$ mission. Table 1 draws together the basic parameters of the star from the literature (Skrutskie et al. 2006; Cutri et al. 2012; Henden et al. 2016; Zacharias et al. 2017). The $K 2$ data reveal that K2-155 is a candidate host of three transiting super-Earths. Systems with multiple planetary candidates are known to have a very low probability of being false positives (FPs; Lissauer et al. 2012). The follow-up observations presented in this paper confirm that the planets are very likely to be genuine.

We organize this paper as follows. Section 2 describes the reduction of the $K 2$ data and detection of the three planet candidates. Section 3 presents follow-up observations using ground-based telescopes, including high-resolution speckle imaging and high-dispersion optical spectroscopy. Section 4 presents our best estimates of the stellar and planetary parameters based on all the data. Section 5 compares K2-155 with another recently discovered planetary system, K2-3, and discusses the potential habitability of the outer planet as well as the prospects for future follow-up observations. Section 6 summarizes all of our findings.

\section{Light Curve Extraction and Transit Search}

K2-155 was observed in the long cadence mode in $K 2$ Campaign 13 from UT 2017 March 8 to 2017 May 27. Our light curve extraction and transit search pipeline were described in detail by Dai et al. (2017) and Livingston et al. (under review). In short, we used the observed motion of the center-oflight on the detector to detrend the systematic flux variation introduced by the rolling motion of the spacecraft, similar to Vanderburg \& Johnson (2014). We searched the detrended light curve (the upper panel of Figure 1) for periodic transit signals with the Box-Least-Squares (BLS) algorithm (Kovács et al. 2002). We found three transiting planet candidates after iteratively searching for the strongest peak in the BLS periodogram and removing the signal of the detected planets. We then scrutinized the light curve and did not see odd-even variations or secondary eclipses, which would be produced by FPs such as a blended eclipsing binary or a hierarchical eclipsing binary.

\section{Observations}

\subsection{Speckle Observations}

We performed high-resolution imaging on the night of UT 2017 September 5 with the WIYN $3.5 \mathrm{~m}$ telescope and the NASA Exoplanet Star and Speckle Imager (NESSI; Scott et al., in preparation). This instrument uses high-speed electronmultiplying CCDs (EMCCDs) to obtain $40 \mathrm{~ms}$ exposures simultaneously in two bands: a "blue" band centered at $562 \mathrm{~nm}$ with a width of $44 \mathrm{~nm}$, and a "red" band centered at $832 \mathrm{~nm}$ with a width of $40 \mathrm{~nm}$. The pixel scales of the 'blue' and "red" EMCCDs are 0!"0175649 pix $^{-1}$ and $0{ }^{\prime \prime} 0181887$ pix $^{-1}$, respectively. We observed K2-155 along with nearby point-source calibrator stars, spaced closely in time. Following the procedures described by Howell et al. (2011), we used the calibrator images to compute reconstructed 256 pix $\times 256$ pix images in each band, corresponding to 4 !" $6 \times 4$ !" 6 .

No additional light sources were detected in the reconstructed images of K2-155. We measured the background sensitivity of the reconstructed images using a series of concentric annuli centered on the target star, resulting in $5 \sigma$ sensitivity limits (in delta-magnitudes) as a function of angular separation. The $5 \sigma$ contrast curve as well as the reconstructed image in each band are displayed in Figure 2.

\subsection{High-dispersion Spectroscopy}

We observed K2-155 with the Tull Coude Spectrograph (Tull et al. 1995) on the McDonald Observatory 2.7 m Harlan J. Smith Telescope on UT 2017 September 14 and 2017 October 14. The spectrograph is a cross-dispersed echelle instrument covering $375-1020 \mathrm{~nm}$, with increasingly larger inter-order gaps longward of $570 \mathrm{~nm}$. A 1.2 arc-second wide slit projects to 2 pixels on the CCD detector, resulting in a spectral resolving power of 60000 . On each date, three successive short exposures were obtained in order to reject cosmic ray events. We used an exposure meter to obtain an accurate flux-weighted barycentric correction, and to establish 

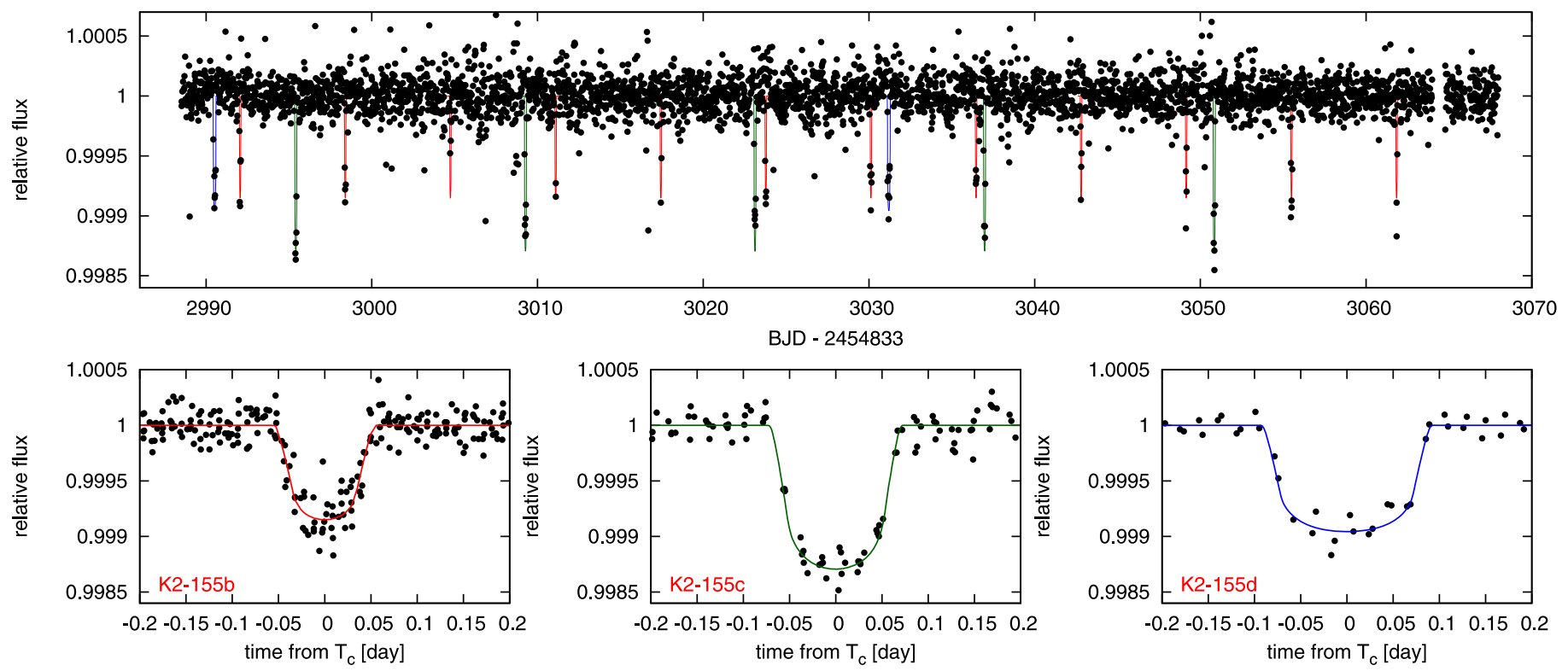

Figure 1. Upper panel: normalized light curve of K2-155 obtained during $K 2$ campaign 13 . The vertical lines indicate the times of planetary transits. Lower panel: folded light curve for each planet.

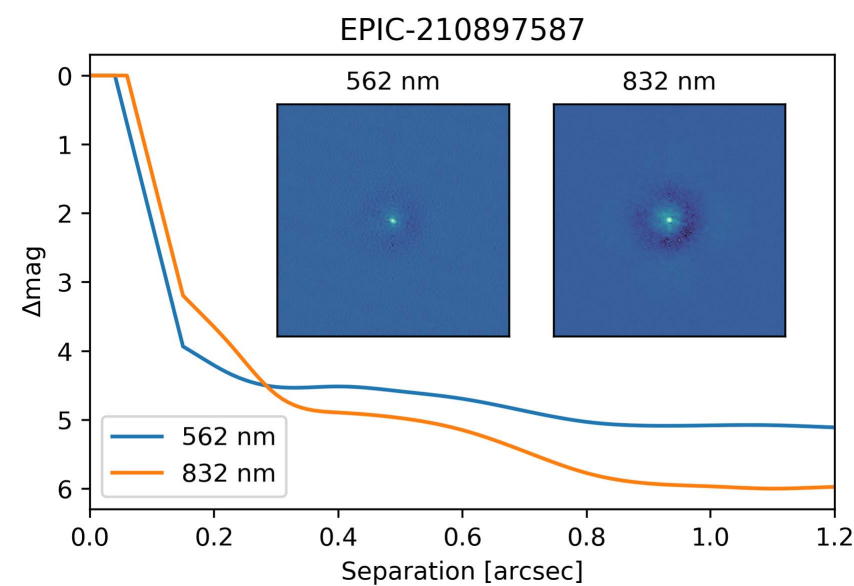

Figure 2. $5 \sigma$ contrast curves of the reconstructed images for K2-155 (insets), based on speckle observations with WIYN/NESSI.

an exposure time resulting in a signal-to-noise ratio $(\mathrm{S} / \mathrm{N})$ of about 30 per pixel. Bracketing exposures of a Th-Ar hollow cathode lamp were obtained in order to generate a wavelength calibration and to remove spectrograph drifts. The raw data were processed using IRAF routines to remove the bias level, inter-order scattered light, and pixel-to-pixel ("flat field") CCD sensitivity variations. We traced the apertures for each spectral order and used an optimal-extraction algorithm to obtain the detected stellar flux as a function of wavelength.

We obtained four high-resolution spectra with the FIbre-fed Echelle Spectrograph (FIES; Frandsen \& Lindberg 1999; Telting et al. 2014) on the $2.56 \mathrm{~m}$ Nordic Optical Telescope (NOT) at the Observatorio del Roque de los Muchachos, La Palma (Spain). The observations were carried out on UT 2017 December 24, 25, 27, and 2018 January 10 as part of the observing programs 2017B/059 (OPTICON) and 56-209 (CAT). We used the 1.!3 high-resolution fiber $(\lambda / \Delta \lambda=$ 67000 ) and set the exposure time to three times 20 minutes, following the same observing strategy as in Gandolfi et al. (2015). We traced the RV drift of the instrument by acquiring
Th-Ar spectra immediately before and after each observation. The data were reduced using standard IRAF and IDL routines. The $\mathrm{S} / \mathrm{N}$ of the extracted spectra is about 20 per pixel at $5500 \AA$.

\section{Data Analysis \\ 4.1. Stellar Parameters}

We analyzed the high-resolution spectra taken by McDonald $2.7 \mathrm{~m} /$ Tull and estimated the stellar parameters. Following Hirano et al. (2017), we used SpecMatch-Emp (Yee et al. 2017) to derive the spectroscopic parameters for K2155. SpecMatch-Emp tries to match the input observed spectrum to hundreds of library spectra covering a wide range of stellar parameters, and finds a subset of stellar spectra that best match the input spectrum. The stellar parameters (the effective temperature $T_{\text {eff }}$, radius $R_{\star}$, and metallicity $\left.[\mathrm{Fe} / \mathrm{H}]\right)$ are then estimated by interpolating the parameters for the bestmatched spectra. We analyzed each of the two Tull spectra separately with SpecMatch-Emp, finding that the results were consistent with each other to within $1 \sigma{ }^{23}$ To check for the accuracy of our analysis, we also applied the same technique to the FIES spectrum and obtained a fully consistent result.

To derive the stellar mass $M_{\star}$, surface gravity $\log g$, density $\rho_{\star}$, and luminosity $L_{\star}$, we used a Monte Carlo technique based on the empirical relations for the stellar parameters of $\mathrm{M}$ dwarfs derived by Mann et al. (2015). Assuming that $T_{\text {eff }}, R_{\star}$, and $[\mathrm{Fe} / \mathrm{H}]$ returned by SpecMatch-Emp follow independent Gaussian distributions, we perturbed those parameters to estimate $M_{\star}, \log g, \rho_{\star}$, and $L_{\star}$ through the absolute $K_{s}$-band magnitude, which we estimated as $5.52 \pm 0.33 \mathrm{mag}$. The result is shown in Table 1, which also includes the distance of

\footnotetext{
23 The library spectra in SpecMatch-Emp were secured by Keck/HIRES. As we discussed in Hirano et al. (2017), we checked the validity of SpecMatch-Emp for the Tull spectra by putting several Tull spectra (mainly $\mathrm{K}$ dwarfs) into the code and found that the output parameters are all consistent with the parameters estimated by the Kea code (Endl \& Cochran 2016) within $2 \sigma$.
} 


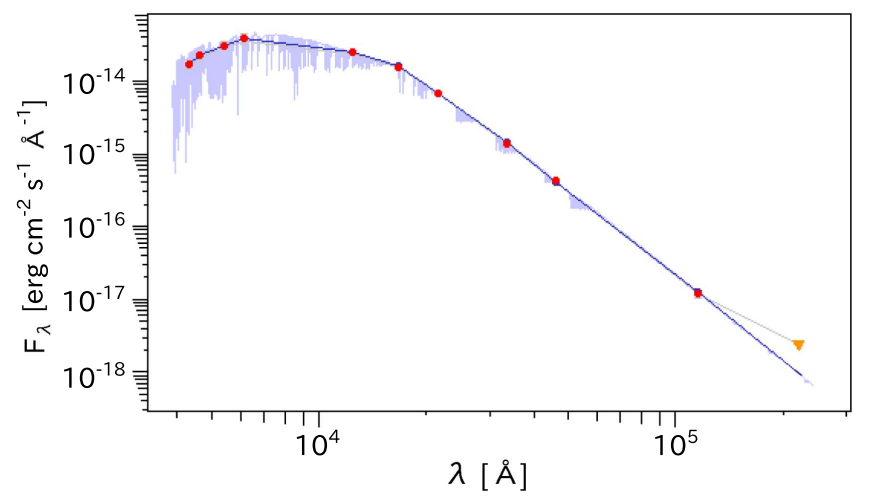

Figure 3. Spectral energy distribution of K2-155. The fluxes based on the magnitudes listed in Table 1 are plotted by the red points. The best-fitting BT-SETTL CIFIST synthetic spectrum is shown in gray. The WISE flux at $22 \mu \mathrm{m}$ (orange triangle) is an upper limit and is not used for the fit.

K2-155, estimated from the absolute and apparent $K_{s}$-band magnitudes.

Following the method described in Gandolfi et al. (2008), we derived the interstellar reddening along the line of sight $\left(A_{\mathrm{v}}\right)$ and obtained an independent estimate of $T_{\text {eff }}$ and $\log g$ for K2155. Briefly, we built the spectral energy distribution (SED) of the star using the APASS $B, g^{\prime}, V, r^{\prime}, 2$ MASS $J, H, K_{\mathrm{s}}$, and WISE $W 1, W 2, W 3$ magnitudes listed in Table 1.

We retrieved the Johnson $B V$, Sloan $g^{\prime}$ and $r^{\prime}$, 2MASS $J H K_{s}$, WISE $W 1, W 2, W 3$, and $W 4$ transmission curves, and absolute flux calibration constants from the Asiago Database on Photometric Systems (Moro \& Munari 2000; Fiorucci \& Munari 2002) and from Wright et al. (2010). We simultaneously fitted the SED for $T_{\text {eff }}$ and $A_{\mathrm{v}}$ using the BT-SETTL CIFIST synthetic spectra from Baraffe et al. (2015). We assumed a total-to-selective extinction of 3.1 (normal interstellar extinction) and adopted the reddening law from Cardelli et al. (1989). We found a reddening of $A_{\mathrm{v}}=0.095 \pm$ $0.050 \mathrm{mag}$, effective temperature of $T_{\text {eff }}=4200 \pm 200 \mathrm{~K}$, and surface gravity of $\log g=5.5 \pm 1.0$ (cgs). The result of the SED fit is shown in Figure 3. Both $T_{\text {eff }}$ and $\log g$ are in good agreement with the spectroscopically derived values, corroborating our results. Note that $A_{\mathrm{v}}$ is explored in the positive range, and thus its estimate could be biased toward higher values.

\subsection{RV Measurements and Star's Membership}

In order to estimate the absolute radial velocities (RVs) of the star and check for any secondary lines in the highresolution spectra, we cross-correlated the Tull spectra against the M2 numerical mask (e.g., Bonfils et al. 2013), developed for the precise RV measurement for HARPS-like spectrographs. To take into account the possible wavelength drift of the spectrograph within the night, we also cross-correlated the spectral segment including strong telluric absorptions (6860-6930 ^) against a theoretical telluric template created by the line-by-line radiative transfer model (LBLRTM; Clough et al. 2005). The absolute RV of K2-155 was calculated by subtracting the telluric RV value (whose magnitude is $\sim 0.5 \mathrm{~km} \mathrm{~s}^{-1}$ ) from the stellar RV value, both of which were estimated by inspecting the peaks of the cross-correlation functions (CCFs).

Table 2 lists the absolute RVs measured from the Tull spectra. The mean absolute RV $\left(19.34 \pm 0.16 \mathrm{~km} \mathrm{~s}^{-1}\right)$ by Tull

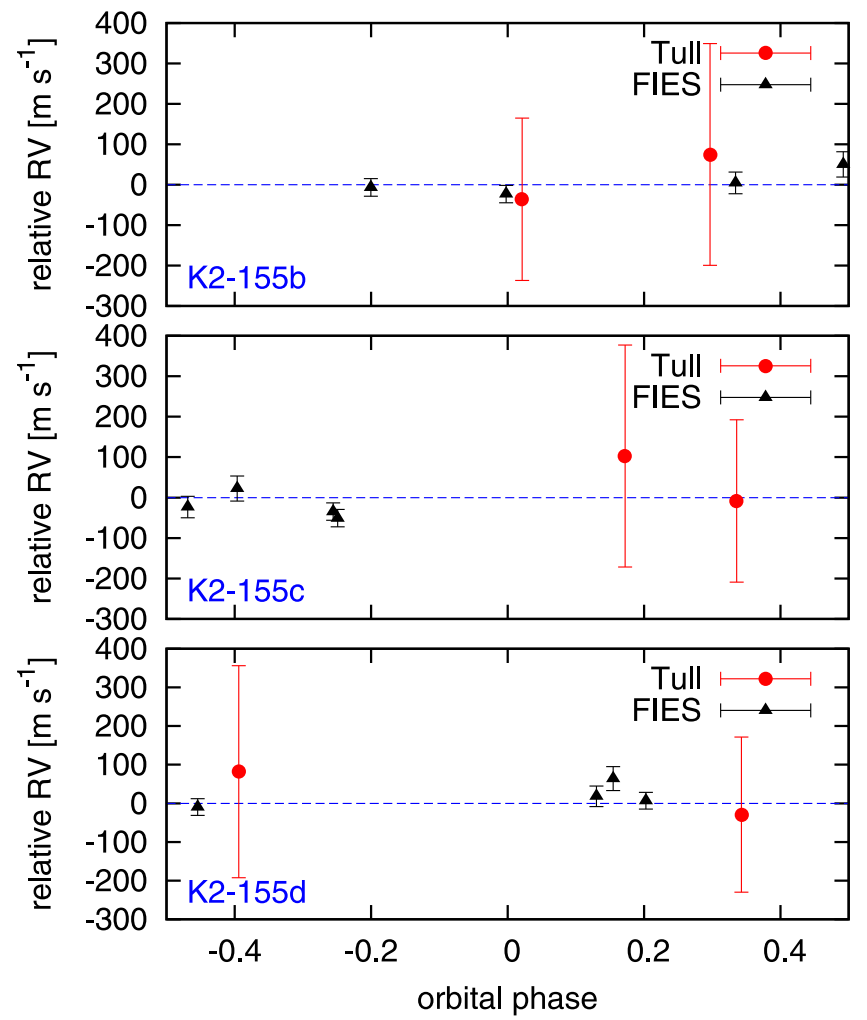

Figure 4. Relative RVs measured by Tull (red circles) and FIES (black triangle), folded by the orbital periods of inner (top), middle (middle), and outer (bottom) planets, respectively.

Table 2

Results of RV Measurements

\begin{tabular}{lccll}
\hline \hline $\begin{array}{l}\text { BJD } \\
(-2450000.0)\end{array}$ & $\begin{array}{c}\text { RV } \\
\left(\mathrm{km} \mathrm{s}^{-1}\right)\end{array}$ & $\begin{array}{c}\text { RV Error } \\
\left(\mathrm{km} \mathrm{s}^{-1}\right)\end{array}$ & RV Type & Instrument \\
\hline 8010.907364 & 19.416 & 0.274 & absolute & Tull \\
8040.879045 & 19.305 & 0.201 & absolute & Tull \\
8112.545268 & 0.000 & 0.027 & relative & FIES \\
8113.547023 & -0.046 & 0.031 & relative & FIES \\
8115.497963 & -0.011 & 0.022 & relative & FIES \\
8129.443988 & -0.028 & 0.022 & relative & FIES \\
\hline
\end{tabular}

is consistent with the value reported in the literature within $2 \sigma$ $\left(20.3 \pm 0.5 \mathrm{~km} \mathrm{~s}^{-1}\right.$; Kharchenko et al. 2007), which also suggests that there has been no significant RV variation of the star over the course of $\sim 10$ years.

For the FIES spectra, we measured relative RVs using multiorder cross-correlations. In doing so, we first derived the RVs by cross-correlating the spectra against the first spectrum. We then applied the RV shift and co-added the individual spectra to obtain the combined spectrum. Finally, the co-added spectrum is used to extract the final RVs. Thus, derived relative RVs are listed in Table 2.

To place an upper limit on the mass of any companion, we estimated the upper limit of the RV semi-amplitude $K$ by fitting the data folded by the orbital periods of the planet candidates. In the fit, we introduced an RV offset parameter for each of the two data sets. This yielded $K=-8 \pm 19 \mathrm{~m} \mathrm{~s}^{-1}$, $K=-38_{-42}^{+39} \mathrm{~m} \mathrm{~s}^{-1}$, and $K=-26_{-25}^{+23} \mathrm{~m} \mathrm{~s}^{-1}$ for the inner, middle, and outer planet candidates, respectively, indicating that the observed RVs are consistent with $K=0 \mathrm{~m} \mathrm{~s}^{-1}$ within 
$\sim 1-\sigma$ for all three periods (Figure 4 ). Thus, the eclipsing binary (EB) scenario for the three planet candidates is strongly constrained. The $2 \sigma$ upper limits on $K$ translate to the upper limits on the companion's mass of $60 M_{\oplus}, 102 M_{\oplus}$, and $75 M_{\oplus}$, respectively, all of which fall on the planetary regime. As the RV data should be fitted for the three companions simultaneously, however, these cannot be interpreted as the mass upper limits of the planet candidates.

The absence of secondary lines in the CCF for Tull spectra also allows us to place an upper limit on the brightness of any close-orbiting companions. To do so, we fitted the observed CCFs by two components: (1) the observed CCF after flattening the continuum to its average value, and (2) the scaled and Doppler-shifted version of the same CCF to mimic a possible faint companion. Here, we implicitly assume that the spectrum of the hypothetical companion is similar to that of the primary (i.e., a late-type star). For a given Doppler shift for the secondary line (relative RV $>15 \mathrm{~km} \mathrm{~s}^{-1}$ ), we computed the possible contamination of a secondary peak, and looked for the maximum contamination flux from a hypothetical companion. We conclude that the contamination is no more than $2 \%$ of the primary star's flux in the visible band, which corresponds to the lowest mass stars $\left(\sim 0.1 M_{\odot}\right)$ for the case of $\mathrm{K} 2-155$. This is a good constraint on the presence of close-in companion(s), but when the companion has a long orbital distance, the relative RV between the primary and secondary stars becomes small (relative $\mathrm{RV} \lesssim 10 \mathrm{~km} \mathrm{~s}^{-1}$ ), and we are not capable of constraining its flux by the present analysis.

The coordinates of K2-155 place it near the same line of sight as the Hyades open cluster. However, K2-155 does not share the same metallicity, proper motion, or radial velocity as typical Hyades stars. The metallicity and mean proper motion of the Hyades are reported to be $[\mathrm{Fe} / \mathrm{H}]=0.14 \pm 0.05$ (Perryman et al. 1998) and $\mu_{\alpha} \cos \delta=1.4 \pm 3.7 \mathrm{mas} \mathrm{yr}^{-1}$ and $\mu_{\delta}=$ $4.3 \pm 4.4$ mas yr$^{-1}$ (Dias et al. 2014), respectively. Together with the absolute $\mathrm{RV},{ }^{24}$ we conclude that $\mathrm{K} 2-155$ is in the background of the Hyades.

Based on the coordinates, proper motion, distance, and RV of $\mathrm{K} 2-155$, we also computed the galactic space velocity $(U, V$, $W$ ) to the Local Standard of Rest (LSR) as in Table 1. The space velocity components are in agreement with those of both thick disk and thin disk stellar populations (e.g., Fuhrmann 2004), making it impossible to tell on this basis to which population K2-155 belongs. The low stellar metallicity is more consistent with the thick disk.

\subsection{Planetary Parameters}

To determine the planetary parameters, we compared two available light curves: our own light curve as produced in Section 2 and the publicly available light curve provided by Vanderburg \& Johnson (2014). The two light curves have almost the same noise level, although our light curve exhibits a slightly larger scatter at the beginning of the $K 2$ observation. We decided to adopt the light curve of Vanderburg \& Johnson (2014) for subsequent analysis.

The fitting procedure of the K2 light curve was described in detail by Hirano et al. (2015), which we summarize here. We first split the light curve into chunks each spanning approximately 5 days and fitted each chunk after removing

\footnotetext{
24 The averaged absolute RV of Hyades members are reported to be $39.29 \pm 0.25 \mathrm{~km} \mathrm{~s}^{-1}$ (Dias et al. 2002).
}

transit signals with a fifth-order polynomial to detrend and obtain the normalized light curve. Then, based on the preliminary ephemerides obtained in Section 2, we extracted small segments of the normalized light curve, which cover the transits of each planet candidate as well as the flux baselines on both sides spanning 2.0 times the transit durations.

For each planet candidate, we simultaneously fitted all of the segments to estimate the global parameters common to all of the segments as well as segment-specific parameters. The global parameters are the scaled semimajor axis $a / R_{\star}$, transit impact parameter $b$, limb-darkening parameters for the quadratic law $\left(u_{1}+u_{2}\right.$ and $\left.u_{1}-u_{2}\right)$, orbital eccentricity and argument of periastron ( $e \cos \omega$ and $e \sin \omega$ ), and planet-to-star radius ratio $R_{p} / R_{\star}$. To take into account possible transit timing variations (TTVs), we allowed the mid-transit time $T_{c}$ to float freely for each light curve segment. We also introduced additional parameters describing the baseline flux variation for each segment, which we assumed to be a linear function of time.

The goodness of fit was assessed with the $\chi^{2}$ statistic:

$$
\chi^{2}=\sum_{i} \frac{\left(f_{\mathrm{obs}, i}-f_{\mathrm{calc}, i}\right)^{2}}{\sigma_{i}^{2}},
$$

where $f_{\text {obs }, i}$ and $f_{\text {calc, } i}$ are the observed and calculated flux, and $\sigma_{i}$ is the flux uncertainty. For the transit model, we integrated the analytic light curve model of Ohta et al. (2009) over the 30 -minute averaging interval of $K 2$ observations. We sampled the posterior distributions of the parameters using our implementation of the Markov Chain Monte Carlo (MCMC) technique (Hirano et al. 2015). In the code, all of the free parameters are first optimized simultaneously by Powell's conjugate direction method (e.g., Press et al. 1992), and the flux baseline parameters are held fixed at the best-fitting values. We then took $10^{6}$ MCMC steps for each planet candidate with all of the parameters being allowed to adjust. We imposed prior distributions for $u_{1}+u_{2}$ and $u_{1}-u_{2}$ adopted from the table by Claret et al. (2013), assuming Gaussian functions with widths of 0.20. Because close-in planets in multi-planet systems are known to have low eccentricities (Van Eylen \& Albrecht 2015), we also imposed Gaussian priors on $e \cos \omega$ and $e \sin \omega$ with their centers and widths being 0 and 0.05 , respectively. For the other parameters, we assumed uniform priors. The reported parameter values and $\pm 1 \sigma$ errors are based on the $50,15.87$, and 84.13 percentile levels of the marginalized posterior distributions. Table 3 gives the results.

Based on the mid-transit times, we calculated the ephemerides $\left(P\right.$ and $\left.T_{c, 0}\right)$ for each planet under the assumption of a constant period. Figure 5 shows the observed minus calculated $(O-C) T_{c}$ plots for the three candidates. The period ratio between $\mathrm{K} 2-155 \mathrm{~b}$ and $\mathrm{K} 2-155 \mathrm{c}$ is somewhat close to $1: 2$, but Figure 5 exhibits no clear sign of TTVs. In the bottom panels of Figure 1, we display the folded transits along with the model light curves (solid lines) based on the parameters given in Table 3 .

\subsection{Validating Planets}

As K2-155 has three planet candidates, the probability that any of the candidates will turn out to be an FP is extremely low. Lissauer et al. (2012) calculated the odds that the systems of multiple transiting planet candidates are FPs. For three-planet 
Table 3

Fitting and Planetary Parameters

\begin{tabular}{|c|c|}
\hline Parameter & Value \\
\hline \multicolumn{2}{|l|}{$K 2-155 b$} \\
\hline$P$ (days) & $6.34365 \pm 0.00028$ \\
\hline$T_{c, 0}(\mathrm{BJD}-2454833)$ & $2985.7153 \pm 0.0021$ \\
\hline$a / R_{\star}$ & $20.3_{-6.1}^{+3.2}$ \\
\hline$b$ & $0.50_{-0.33}^{+0.30}$ \\
\hline$R_{p} / R_{\star}$ & $0.0271_{-0.0012}^{+0.0023}$ \\
\hline$u_{1}$ & $0.36 \pm 0.13$ \\
\hline$u_{2}$ & $0.41 \pm 0.14$ \\
\hline$R_{p}\left(R_{\oplus}\right)$ & $1.55_{-0.17}^{+0.20}$ \\
\hline$a(\mathrm{au})$ & $0.0546 \pm 0.0019$ \\
\hline$S_{p}\left(S_{\oplus}\right)$ & $19.9 \pm 4.5$ \\
\hline \multicolumn{2}{|l|}{$K 2-155 c$} \\
\hline$P$ (days) & $13.85402 \pm 0.00088$ \\
\hline$T_{c, 0}(\mathrm{BJD}-2454833)$ & $2981.5643 \pm 0.0025$ \\
\hline$a / R_{\star}$ & $30.0_{-9.8}^{+6.2}$ \\
\hline$b$ & $0.57_{-0.39}^{+0.27}$ \\
\hline$R_{p} / R_{\star}$ & $0.0339_{-0.0016}^{+0.0031}$ \\
\hline$u_{1}$ & $0.33 \pm 0.13$ \\
\hline$u_{2}$ & $0.40_{-0.14}^{+0.13}$ \\
\hline$R_{p}\left(R_{\oplus}\right)$ & $1.95_{-0.22}^{+0.27}$ \\
\hline$a(\mathrm{au})$ & $0.0920 \pm 0.0032$ \\
\hline$S_{p}\left(S_{\oplus}\right)$ & $7.0 \pm 1.6$ \\
\hline
\end{tabular}

\section{$K 2-155 d$}

$P$ (days)

$T_{c, 0}(\mathrm{BJD}-2454833)$

$a / R_{\star}$

$b$

$R_{p} / R_{\star}$

$u_{1}$

$u_{2}$

$R_{p}\left(R_{\oplus}\right)$

$a$ (au)

$S_{p}\left(S_{\oplus}\right)$
$40.6835 \pm 0.0031$

$2949.8324 \pm 0.0048$ $73.5_{-15.9}^{+8.4}$ $0.41_{-0.28}^{+0.30}$ $0.0286_{-0.0010}^{+0.0015}$

$0.32 \pm 0.12$ $0.38_{-0.13}^{+0.14}$ $1.64_{-0.17}^{+0.18}$

$0.1886 \pm 0.0066$ $1.67 \pm 0.38$

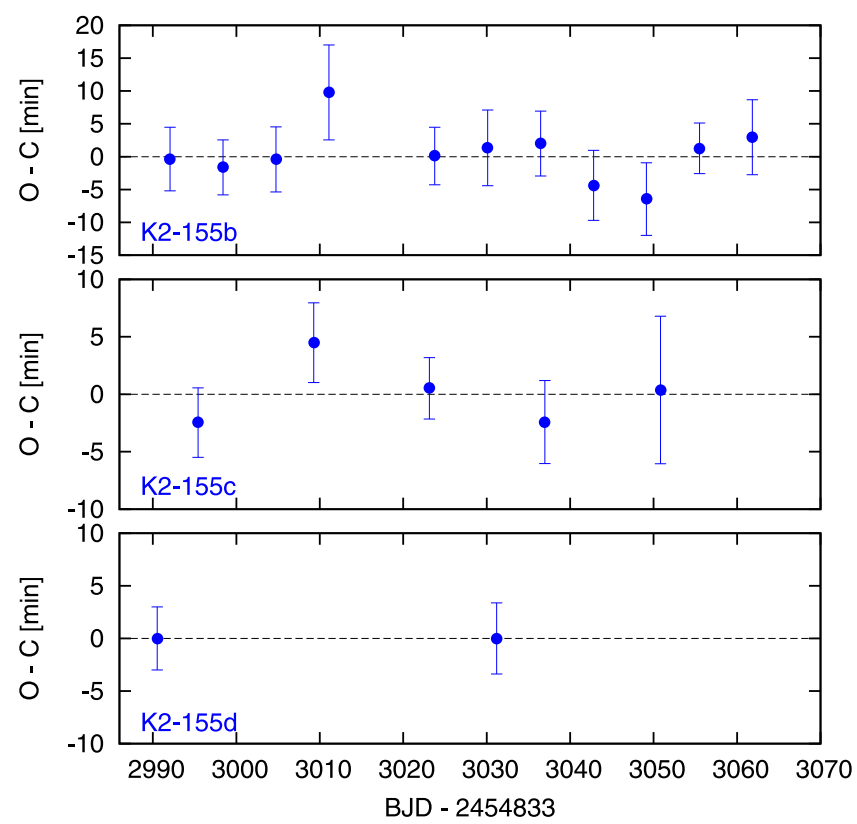

Figure 5. $O-C$ diagrams for the mid-transit times $T_{c}$. There is no evidence of significant TTVs.

systems, they found that fewer than one such system is expected to contain an FP among the entire Kepler sample. In this sense, the presence of three candidates is self-validating. Below, we investigate the constraints on FP scenarios based on direct follow-up observations rather than the statistical argument of Lissauer et al. (2012).

As shown in Section 4.2, the absence of a large RV variation $\left(\gtrsim 100 \mathrm{~m} \mathrm{~s}^{-1}\right)$ as well as a secondary peak in the CCFs implied that the transit signals are not caused by a stellar companion orbiting and occulting K2-155 (i.e., EB). The remaining possible FP scenarios are background eclipsing binaries (BEB) and hierarchical-triple eclipsing binaries (HEB). However, these scenarios are also constrained by the lack of bright nearby sources in the reconstructed image from the speckle observations (Figure 2). In addition, checking the POSS1 archival images taken in 1950 (Figure 6), we found no bright star at the current position of K2-155, verifying that no background sources are hidden in the reconstructed image of K2-155 by chance alignment.

As the speckle observations with WIYN/NESSI are only able to find companions in the proximity of the target, it is still possible that a fainter object at a large separation is blended in the $K 2$ aperture, which could be responsible for the transit-like photometric signals. We thus searched for fainter objects within $20^{\prime \prime}$ from K2-155 using the SDSS photometric catalog (Alam et al. 2015). As a consequence, we identified five stars within $12^{\prime \prime}-20^{\prime \prime}$ from K2-155, but all of those stars have $r$-band magnitudes (similar to the Kepler magnitudes) fainter than $20 \mathrm{mag}$. The $r$-magnitude of $\mathrm{K} 2-155$ is $12.437 \pm 0.002 \mathrm{mag}$, and thus the maximum magnitude that can produce an eclipse depth of $0.1 \%$ is $r=19.9 \mathrm{mag}$ ( $100 \%$ occultation). Therefore, we conclude that K2-155 is the source of the transit signals.

Regarding the HEB scenario, the speckle observations achieved a $5 \sigma$ contrast of $4.2 \mathrm{mag}(562 \mathrm{~nm})$ at 0.2 , corresponding to the mass upper limit of $\approx 0.1 M_{\odot}$ for a possible bound companion (e.g., Dotter et al. 2008) at the projected separation of $\approx 12 \mathrm{au}$ and further. There is still a possibility, however, that a very late-type star is orbiting K2-155 at an orbital distance of 1-12 au; for instance, a $0.1 M_{\odot}$ star with $P=2$ year exerts an RV semi-amplitude of only $\approx 3 \mathrm{~km} \mathrm{~s}^{-1}$, which could be overlooked in the RV data (Table 2). But even if this is the case and the bound later-type star is responsible for the transit signals, the depths of these candidates correspond to those of "planets."

The fact that K2-155 is transited by the three planet candidates is corroborated by comparing the mean stellar density inferred from spectroscopy $\left(\rho_{\star}=3.84 \pm 0.79 \rho_{\odot}\right)$ with the mean stellar density implied by the transit modeling. The scaled semimajor axes $a / R_{\star}$ in Table 3 are translated into the mean stellar densities of $2.8_{-1.8}^{+1.6} \rho_{\odot}$ for the inner, $1.9_{-1.3}^{+1.4} \rho_{\odot}$ for the middle, and $3.2_{-1.7}^{+1.2} \rho_{\odot}$ for the outer planet, respectively. Hence, the stellar densities estimated from transit modelings are consistent with the spectroscopic density for K2-155 within about $1 \sigma$, but would be inconsistent with later-type stars; according the observed mass-radius relation for $\mathrm{M}$ dwarfs (e.g., Mann et al. 2015), the mean density of mid-to-late $M$ dwarfs with $M_{\star}<0.4 M_{\odot}$ is higher than $\approx 6 \rho_{\odot}$.

To quantify the false positive probability (FPP) of each planet candidate, we used the statistical framework implemented in the vespa software package (Morton 2012, 2015). This code simulates FP scenarios using the TRILEGAL Galaxy model (Girardi et al. 2005) and assesses the likelihoods of EB, 

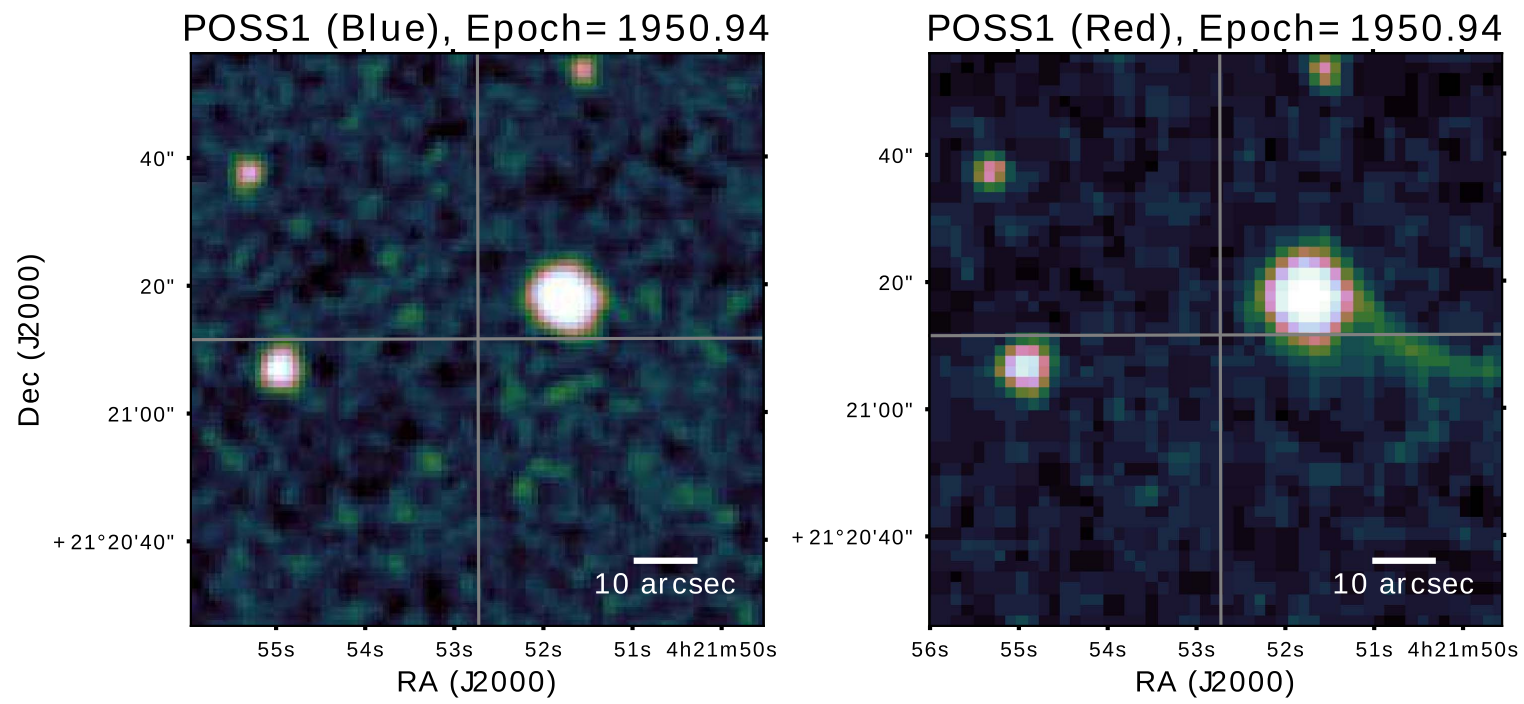

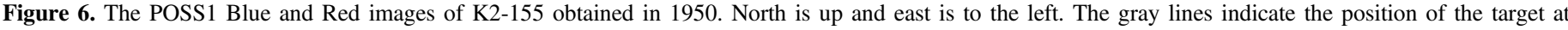

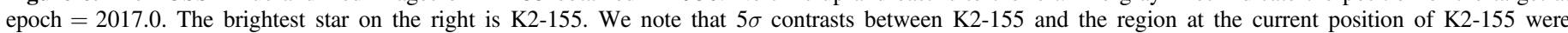
5-5.5 mag.

Table 4

Comparison between the K2-155 System and K2-3 System

\begin{tabular}{|c|c|c|c|c|c|c|c|}
\hline Planet & $P$ (days) & $R_{p}\left(R_{\oplus}\right)$ & $S_{p}\left(S_{\oplus}\right)$ & Planet & $P$ (days) & $R_{p}\left(R_{\oplus}\right)$ & $S_{p}\left(S_{\oplus}\right)$ \\
\hline $\mathrm{K} 2-155 \mathrm{~b}$ & $6.34365 \pm 0.00028$ & $1.55_{-0.17}^{+0.20}$ & $19.9 \pm 4.5$ & $\mathrm{~K} 2-3 \mathrm{~b}$ & $10.05403_{-0.00025}^{+0.00026}$ & $1.90 \pm 0.20$ & $8.7 \pm 2.0$ \\
\hline $\mathrm{K} 2-155 \mathrm{c}$ & $13.85402 \pm 0.00088$ & $1.95_{-0.22}^{+0.27}$ & $7.0 \pm 1.6$ & $\mathrm{~K} 2-3 \mathrm{c}$ & $24.6454 \pm 0.0013$ & $1.52 \pm 0.17$ & $2.64 \pm 0.59$ \\
\hline $\mathrm{K} 2-155 \mathrm{~d}$ & $40.6835 \pm 0.0031$ & $1.64_{-0.17}^{+0.18}$ & $1.67 \pm 0.38$ & $\mathrm{~K} 2-3 \mathrm{~d}$ & $44.55612 \pm 0.00021$ & $1.35 \pm 0.16$ & $1.20 \pm 0.27$ \\
\hline
\end{tabular}

$\mathrm{BEB}$, and HEB scenarios. The inputs to vespa are the phasefolded light curve, the size of the photometric aperture, contrast curves from high-resolution imaging, the maximum secondary eclipse depth allowed by the $K 2$ light curve, as well as the broadband photometry and spectroscopic stellar parameters of the host star. The FPPs computed by vespa are below $10^{-5}$ for all three planet candidates of K2-155. However, because vespa considers each planet individually, it does not take into account the "multiplicity boost" suggested by Lissauer et al. (2012), who found that planet candidates belonging to stars with three or more candidates are a priori $\sim 100$ times more likely to be valid planets than single candidates. This means that the FPPs computed by vespa are likely to be overestimated by two orders of magnitude. Each of K2-155's three planet candidates are therefore below the fiducial validation threshold of $1 \%$ FPP by some five orders of magnitude. Thus, all three candidates are quantitatively validated, in addition to our independent determination of the low likelihoods of FP scenarios. We conclude that the three candidates of K2-155 are indeed bona fide planets.

\section{Discussion}

\subsection{Comparison with the K2-3 System}

$\mathrm{K} 2-155$ is similar to K2-3 (Crossfield et al. 2015) in many aspects. Both stars are relatively bright early $M$ dwarfs $(V=12.81 \mathrm{mag}$ for $\mathrm{K} 2-155$ and $V=12.17 \mathrm{mag}$ for $\mathrm{K} 2-3$ ) hosting three transiting super-Earths. Table 4 summarizes the planetary parameters for the two systems (Dai et al. 2016; Fukui et al. 2016; Hirano et al. 2017). In both systems, the outermost transiting planets receive stellar insolations that are slightly higher than the solar insolation on the Earth $\left(1 S_{\oplus}\right)$, but less than $\approx 2 S_{\oplus}$. One difference between the two systems is the size ordering of the planets. For K2-155 the middle planet is the largest, while for K2-3 the inner planet is the largest.

\subsection{Habitability of K2-155d}

The outer planet (K2-155d) has a relatively long orbital period and receives a stellar insolation flux similar to that of Earth $\left(S=1.67 \pm 0.38 S_{\oplus}\right)$. This implies that $\mathrm{K} 2-155 \mathrm{~d}$ is located in or near the habitable zone around K2-155. Another factor affecting potential habitability is whether the planet has a solid surface or is smothered by a massive atmosphere. Rogers (2015) noted that planets larger than $1.6 R_{\oplus}$ are likely to possess volatile-rich atmospheres. The size of K2-155d $\left(\approx 1.64 R_{\oplus}\right)$ is very close to this boundary. It also falls within the observed "valley" in the planet radius distribution (Fulton et al. 2017), making it a particularly interesting target for characterizing its internal structure and atmosphere. Recently, Van Eylen et al. (2017) confirmed the presence of the radius gap with more precise measurements of stellar and thus planetary radii, and found that its dependence on the orbital period suggests that it is likely caused by photoevaporation.

At this point, it is unclear whether $\mathrm{K} 2-155 \mathrm{~d}$ is rocky or not, until we make a precise mass measurement by RV or TTV observations. We decided to investigate whether the planet would be habitable if it does turn out to have an Earth-like composition and atmosphere. Three-dimensional (3D) global climate simulations have shown that tidally locked planets can have a moderate surface temperature in a wide range of orbital distance due to the climate-stabilizing effects of dayside clouds 

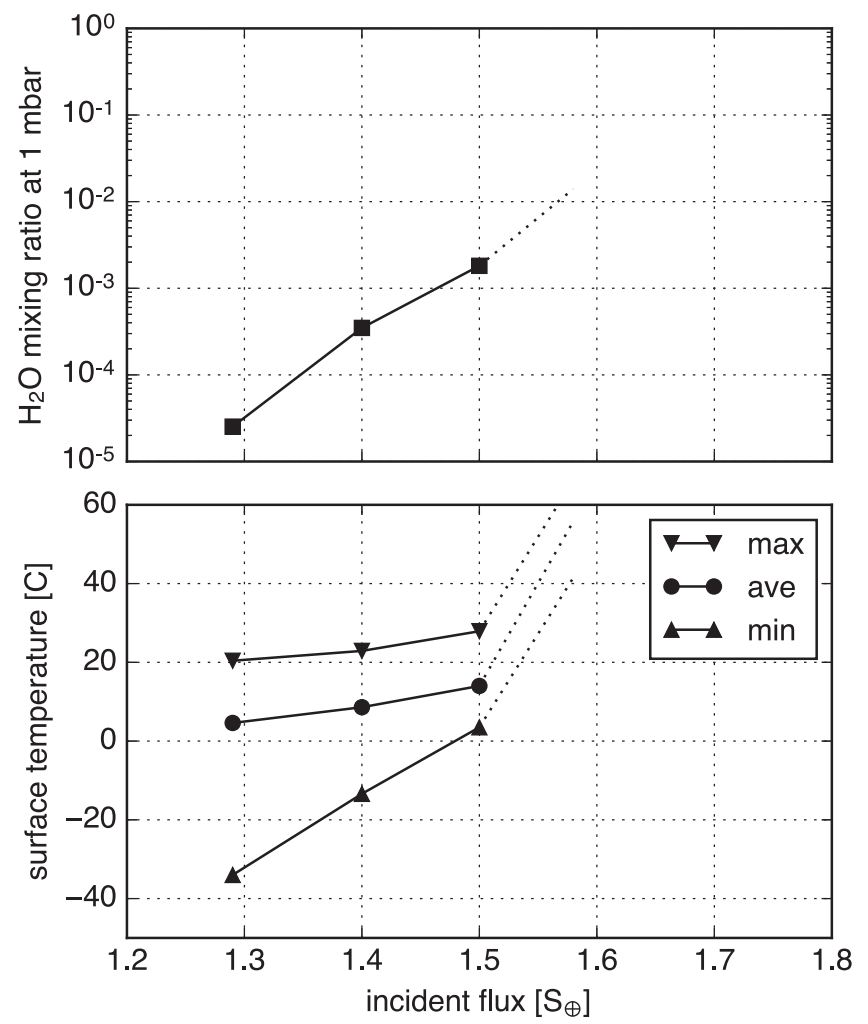

Figure 7. Results of 3D global climate simulations for K2-155d. We plot the water mixing ratio at 1 mbar (upper) and global maximum, average, and minimum surface temperatures (lower) as a function of insolation flux on $\mathrm{K} 2-155 \mathrm{~d}$. When the insolation exceeds $\approx 1.5 S_{\oplus}$, both surface temperature and water mixing ratio continue to increase until they eventually enter the regime where the model is invalid due to high temperature and high humidity.

(Yang et al. 2013; Kopparapu et al. 2016) but may undergo the classical moist greenhouse state at the higher end of the incident flux. Recent studies suggested that for an Earth-sized planet with a nitrogen-dominated atmosphere around an M1 star, this occurs when the total incident flux exceeds $\approx 1.4 S_{\oplus}$ (Fujii et al. 2017; Kopparapu et al. 2017).

In earlier studies, the planet was assumed to be an Earthsized one $\left(1.0 R_{\oplus}\right)$ with the Earth's surface gravity. In order to find a possible climate specifically for K2-155d, we ran a series of global climate simulations using a 3D General Circulation Model ROCKE-3D (Way et al. 2017), fixing the planetary parameters at the values of $\mathrm{K} 2-155 \mathrm{~d}$. The setup is equivalent to the model coupled with a dynamic ocean ( $900 \mathrm{~m}$ depth) used in Fujii et al. (2017), except that the planetary size and the rotation/orbital period are specified for K2-155d. Namely, the planetary radius is fixed at $1.6 R_{\oplus}$ and its mass is set to $4.2 M_{\oplus}$ based on the empirical relation of Weiss \& Marcy (2014). Given the proximity to the star, the three planets around K2155 are expected to be tidally locked (e.g., Kasting et al. 1993; Barnes 2017), and thus the rotation period is assumed to be equal to the orbital period (40.6835 days). For the input stellar spectrum, we adopted the PHOENIX atmosphere model (BTSETTL; Allard et al. 2013) for which we adopted the stellar parameters of K2-155. We assumed the planet is covered with a thermodynamic ocean, and assumed a 1 bar $\mathrm{N}_{2}$ atmosphere and $1 \mathrm{ppm}$ of $\mathrm{CO}_{2}$ as in Fujii et al. (2017). We increased the incident flux from $1.29 S_{\oplus}$ (the $1 \sigma$ lower limit of $S_{p}$ in Table 3) to $1.67 S_{\oplus}$ (the best-fit value) and checked the range that allows for the planet to have a moderate surface temperature. The upper panel of Figure 7 shows the water mixing ratio at 1 mbar for varying incident flux, while the lower panel presents the corresponding maximum, global average, and minimum surface temperatures.

Similarly to previous works for an Earth analogue, equilibrium climates were secured up to $S_{p} \approx 1.5 S_{\oplus}$. Above this limit, the model surface temperature continues to increase until it enters the regime where the model is invalid and the simulation stops. When the insolation is close to or lower than $1.5 S_{\oplus}$, the surface temperature remains moderate, comparable to that of the Earth. The upper humidity increases gradually as incident flux increases and is about to cross the classical moist greenhouse state at about $1.5 S_{\oplus}$. Thus, K2-155d has a potential of being habitable if the incident flux turns out to be close to the lower end within the uncertainty range, though the actual habitability also depends on other factors including its atmosphere, water content, and initial stellar luminosity (e.g., Luger \& Barnes 2015; Tian \& Ida 2015). Another important factor that potentially affects the habitability is the presence of frequent flares of the host star (e.g., Vida et al. 2017). We inspected the $K 2$ light curve of K2-155 but found no such event over the course of 80 days.

\subsection{Prospects on Future Follow-up Observations}

Given the brightness for an M dwarf, K2-155 is an attractive target for future follow-up studies, including Doppler mass measurements and transit photometry. Among $\mathrm{M}$ dwarfs $\left(T_{\text {eff }} \leqslant 4000 \mathrm{~K}\right)$ with transiting planets, K2-155 is the fourth brightest star in the $V$-band, after GJ 436, K2-3, and GJ 3470. It is also the second brightest $\mathrm{M}$ dwarf in optical passbands (after K2-3) having a possibly habitable transiting planet $\left(S_{p} \lesssim 2.0 S_{\oplus}\right)$.

Based on the empirical mass estimates by Weiss \& Marcy (2014), we estimate the RV semi-amplitudes of the planets to be $K \approx 2.1 \mathrm{~m} \mathrm{~s}^{-1}, 2.0 \mathrm{~m} \mathrm{~s}^{-1}$, and $1.2 \mathrm{~m} \mathrm{~s}^{-1}$ for the inner, middle, and outer planets, respectively, suggesting that the masses of at least inner two planets could be constrained by gathering a large number $(\sim 50-100)$ data points with a precision of 2-3 $\mathrm{m} \mathrm{s}^{-1}$ (e.g., Guenther et al. 2017). Although challenging, observations of $\mathrm{M}$ dwarfs of similar magnitude (e.g., K2-3) have shown that RV precisions of 2-3 $\mathrm{m} \mathrm{s}^{-1}$ were achieved by TNG/HARPS-N and Magellan/PSF (e.g., Almenara et al. 2015; Dai et al. 2016), and thus these measurements seem feasible with high precision spectrographs on $8-10 \mathrm{~m}$ telescopes such as Keck/HIRES. Considering that the three planets straddle the rocky to volatile-rich boundary (Rogers 2015) and also the radius gap suggested by Fulton et al. (2017), the comparison between the mean densities of these planets may provide some insight into the origin of closein super-Earths in multi-planet systems.

We note that the expected RV jitter for K2-155 is small. In order to estimate the rotation period of the star, we computed the Lomb-Scargle periodogram and auto-correlation function of the light curve (McQuillan et al. 2014), both of which are shown in Figure 8. Both methods yielded similar estimates for the rotation period $\left(P_{\text {rot }}=47.5_{-9.0}^{+19.3}\right.$ days and $P_{\text {rot }}=46.2_{-5.6}^{+8.1}$ days, respectively), although the detected period could be an alias given the short observing span of $K 2$ ( $\sim 80$ days). Using this tentative rotation period together with the stellar radius $\left(R_{\star}=0.526 R_{\odot}\right)$, we estimate the equatorial velocity of the star as $\approx 0.58 \mathrm{~km} \mathrm{~s}^{-1}$, which is the maximum value for the projected stellar spin velocity $(v \sin i)$. The $K 2$ light curve exhibits the photometric 

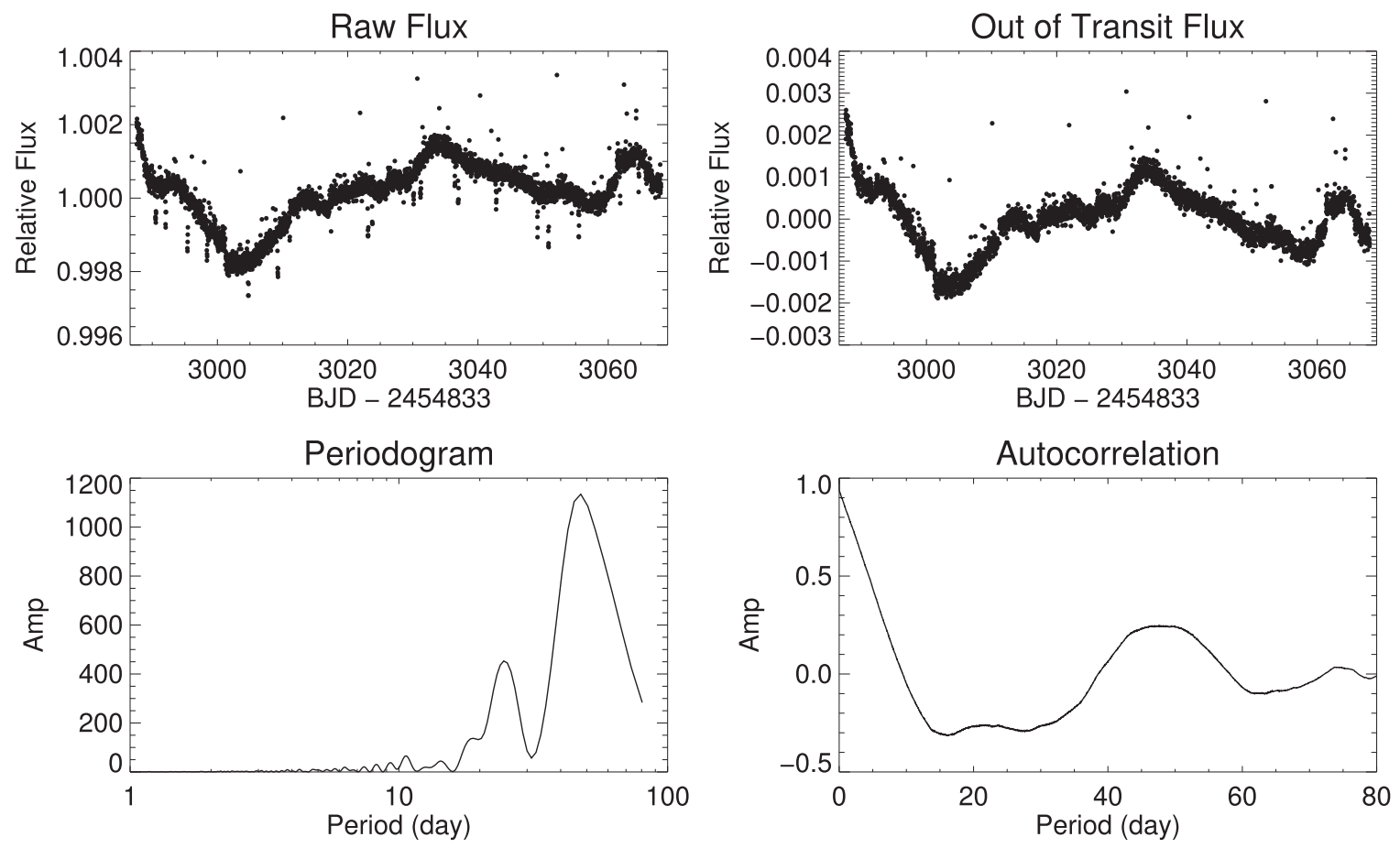

Figure 8. K2-155's light curve before detrending (top two panels), and its Lomb-Scargle periodogram (bottom left) and auto-correlation function (bottom right).

variation amplitude of $\approx 0.2 \%$, and hence the maximum stellar jitter in the visible wavelengths should be no more than $\approx 1 \mathrm{~m} \mathrm{~s}^{-1}$.

Transiting planets with relatively long orbital periods ( $P>30$ days) detected by $K 2$ sometimes suffer from the problem of "stale ephemerides" due to the small number of observed transits. Indeed, only two transits of K2-155d were observed by $K 2$, leading to a large uncertainty in its orbital period. Follow-up transit observations are encouraged to enable accurate long-term predictions of transit times. K2-155 could be a good target for the upcoming CHEOPS space mission (CHaracterizing ExOPlanet Satellite; Broeg et al. 2013), which is specifically designed to observe low-amplitude transits around bright stars. The orbital periods of K2-155b and K2$155 \mathrm{c}$ appear to be close to a 2:1 mean-motion resonance and those of K2-155c and K2-155d are close to a 3:1 resonance, but no clear signs of TTVs were seen in the $O-C$ diagrams (Figure 5). It will be interesting to see if future transit observations reveal any TTVs in this system.

The brightness of K2-155 also facilitates transit spectroscopy as a means of probing the atmospheres of the super-Earths. Following Niraula et al. (2017), we plot in Figure 9 the "relative $\mathrm{S} / \mathrm{N}$ " of transmission spectroscopy for known transiting planets except hot Jupiters $\left(R_{p}<6.0 R_{\oplus}\right)$ around $\mathrm{M}$ dwarfs, based on the stellar and planet radii, atmospheric scale height, $V$-band magnitude, and transit duration (see Equation 1(b)) and (b) of Niraula et al. 2017). We here plotted the $\mathrm{S} / \mathrm{N}$ per transit rather than the $\mathrm{S} / \mathrm{N}$ for a given period of time as in Niraula et al. (2017). The three planets around K2-155 are plotted with the colored circles. Many Neptune-class planets $\left(R_{p}=2.0-6.0 R_{\oplus} ;\right.$ according to Kepler's classification) show a higher $\mathrm{S} / \mathrm{N}$, but among super-Earths and Earth-like planets $\left(R_{p}<2.0 R_{\oplus}\right), \mathrm{K} 2-155 \mathrm{c}$ is one of the best targets for transmission spectroscopy. Figure 9 also shows that K2-155d

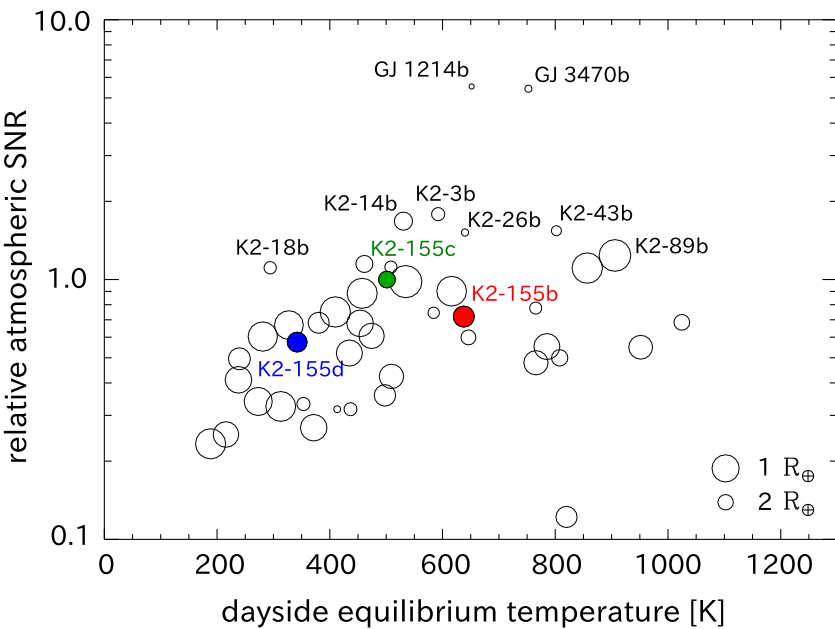

Figure 9. Relative $\mathrm{S} / \mathrm{N}$ of transmission spectroscopy for known transiting planets $\left(R_{p}<6.0 R_{\oplus}\right)$ around $\mathrm{M}$ dwarfs, calculated based on the stellar and planet radii, atmospheric scale height, $V$-band magnitude, and transit duration. The S/N's for K2-155b, c, and d are plotted with the red, green, and blue circles, respectively.

is a good target in the sample as a possibly habitable superEarth.

The actual signal amplitudes of the three super-Earths depend on the (unknown) scale heights of their atmospheres. The atmospheric feature in transmission spectroscopy is of the order of $10 H \cdot R_{p} / R_{\star}^{2}$, where $H$ is the atmospheric scale height (Miller-Ricci et al. 2009). When a cloud-free hydrogendominated atmosphere is assumed, the variation amplitude in transit depth is expected to be $60 \mathrm{ppm}$ (K2-155d) to $120 \mathrm{ppm}$ (K2-155c), which would be detectable by observations from the space (e.g., Hubble Space Telescope). But if the planets have an Earth-like atmosphere (i.e., mean molecular weight of 
$\mu \sim 30$ ), the expected signal would be $4-8 \mathrm{ppm}$ and its detection would be challenging.

\section{Summary}

In this paper, we have identified $\mathrm{K} 2-155$, a relatively bright M dwarf observed in the $K 2$ Campaign field 13, as a candidate planetary system with three transiting super-Earths, and validated all of these planets based on speckle imaging and high-resolution spectroscopy. The coordinates of K2-155 are similar to that of the Hyades cluster, but our spectroscopy indicates that its metallicity $([\mathrm{Fe} / \mathrm{H}]=-0.42 \pm 0.12)$ is too low for a Hyades member, and the RV and proper motions are also inconsistent with those of Hyades members. Indeed, K2155 is one of the most metal-poor M-dwarf planet hosts, which along with its long rotation period $(\approx 46$ days) suggests that it is significantly older than the Hyades.

$\mathrm{K} 2-155 \mathrm{~d}$ resides in or near the habitable zone, which led us to perform three-dimensional (3D) global climate simulations to estimate the surface temperature of K2-155d assuming that the planet has an Earth-like composition and atmosphere. We found that if the stellar insolation on K2-155d is smaller than $1.5 S_{\oplus}$, the planet could maintain a moderate climate with the averaged surface temperatures of $\lesssim 20^{\circ} \mathrm{C}$ and a stratospheric water vapor mixing ratio comparable to or below the classical moist greenhouse limit. The stellar insolation on $\mathrm{K} 2-155 \mathrm{~d}$ has a large uncertainty $\left(S_{p}=1.67 \pm 0.38 S_{\oplus}\right)$ and thus its actual habitability is not known at this point, but given the brightness of the host star, this possibly habitable planet as well as the inner two planets in this system are good targets for future follow-up studies including Doppler mass measurements and transmission spectroscopy.

We thank Adrian Price-Whelan for advice on stellar kinematics. We thank the NOT staff members, in particular Peter Sørensen, for their help, and support during the observations. Based on observations made with the Nordic Optical Telescope, operated by the Nordic Optical Telescope Scientific Association at the Observatorio del Roque de los Muchachos, La Palma, Spain, of the Instituto de Astrofisica de Canarias. Data presented herein were obtained at the WIYN Observatory from telescope time allocated to NN-EXPLORE through the scientific partnership of the National Aeronautics and Space Administration, the National Science Foundation, and the National Optical Astronomy Observatory, obtained as part of an approved NOAO observing program (P.I. Livingston, proposal ID 2017B-0334). NESSI was built at the Ames Research Center by Steve B. Howell, Nic Scott, Elliott P. Horch, and Emmett Quigley. This work was supported by Japan Society for Promotion of Science (JSPS) KAKENHI grant No. JP16K17660. D.G. gratefully acknowledges the financial support of the Programma Giovani Ricercatori-Rita Levi Montalcini-Rientro dei Cervelli (2012) awarded by the Italian Ministry of Education, Universities and Research (MIUR). This project has received funding from the European Union's Horizon 2020 research and innovation programme under grant agreement No. 730890. This material reflects only the authors views and the Commission is not liable for any use that may be made of the information contained therein. I.R. acknowledges support by the Spanish Ministry of Economy and Competitiveness (MINECO) and the Fondo Europeo de Desarrollo Regional (FEDER) through grant ESP2016-80435C2-1-R, as well as the support of the Generalitat de Catalunya/
CERCA program. M.E. and W.D.C. were supported by NASA grant NNX16AJ11G to The University of Texas at Austin. The authors are honored to be permitted to conduct observations on Iolkam Du'ag (Kitt Peak), a mountain within the Tohono O'odham Nation with particular significance to the Tohono O'odham people.

Software: IRAF (Tody 1986, 1993), SpecMatch-Emp (Yee et al. 2017), PHOENIX (Allard et al. 2013), vespa (Morton 2012, 2015) ROCKE-3D (Way et al. 2017).

\section{ORCID iDs}

Teruyuki Hirano (iD https://orcid.org/0000-0003-3618-7535

Fei Dai (iD https://orcid.org/0000-0002-8958-0683

William D. Cochran (iD https://orcid.org/0000-00019662-3496

Michael Endl (ib https://orcid.org/0000-0002-7714-6310

Davide Gandolfi (10 https://orcid.org/0000-0001-8627-9628

Seth Redfield (i) https://orcid.org/0000-0003-3786-3486

Joshua N. Winn (DD https://orcid.org/0000-0002-4265-047X

Juan Cabrera (iD https://orcid.org/0000-0001-6653-5487

Hans Deeg (1) https://orcid.org/0000-0003-0047-4241

Malcolm Fridlund (ib https://orcid.org/0000-0003-2180-9936

Akihiko Fukui (D) https://orcid.org/0000-0002-4909-5763

Sascha Grziwa (1D https://orcid.org/0000-0003-3370-4058

Norio Narita (ib https://orcid.org/0000-0001-8511-2981

Grzegorz Nowak (i) https://orcid.org/0000-0002-7031-7754

Ignasi Ribas (iD https://orcid.org/0000-0002-6689-0312

\section{References}

Alam, S., Albareti, F. D., Allende Prieto, C., et al. 2015, ApJS, 219, 12 Allard, F., Homeier, D., Freytag, B., et al. 2013, MSAIS, 24, 128 Almenara, J. M., Astudillo-Defru, N., Bonfils, X., et al. 2015, A\&A, 581, L7 Baraffe, I., Homeier, D., Allard, F., \& Chabrier, G. 2015, A\&A, 577, A42 Barnes, R. 2017, CeMDA, 129, 509

Berta-Thompson, Z. K., Irwin, J., Charbonneau, D., et al. 2015, Natur, 527, 204

Bonfils, X., Delfosse, X., Udry, S., et al. 2013, A\&A, 549, A109

Bonfils, X., Gillon, M., Udry, S., et al. 2012, A\&A, 546, A27

Broeg, C., Fortier, A., Ehrenreich, D., et al. 2013, European Physical Journal Web of Conferences, 47, 03005

Butler, R. P., Vogt, S. S., Marcy, G. W., et al. 2004, ApJ, 617, 580

Cardelli, J. A., Clayton, G. C., \& Mathis, J. S. 1989, ApJ, 345, 245

Claret, A., Hauschildt, P. H., \& Witte, S. 2013, A\&A, 552, A16

Clough, S. A., Shephard, M. W., Mlawer, E. J., et al. 2005, JQSRT, 91, 233

Crossfield, I. J. M., Petigura, E., Schlieder, J. E., et al. 2015, ApJ, 804, 10

Cutri, R. M., Wright, E. L., Conrow, T., et al. 2012, yCat, 2311, 0

Dai, F., Winn, J. N., Albrecht, S., et al. 2016, ApJ, 823, 115

Dai, F., Winn, J. N., Gandolfi, D., et al. 2017, AJ, 154, 226

Dias, W. S., Alessi, B. S., Moitinho, A., \& Lépine, J. R. D. 2002, A\&A, 389,871

Dias, W. S., Monteiro, H., Caetano, T. C., et al. 2014, A\&A, 564, A79

Dotter, A., Chaboyer, B., Jevremović, D., et al. 2008, ApJS, 178, 89

Dressing, C. D., Vanderburg, A., Schlieder, J. E., et al. 2017, AJ, 154, 207

Endl, M., \& Cochran, W. D. 2016, PASP, 128, 094502

Fiorucci, M., \& Munari, U. 2002, Ap\&SS, 280, 77

Frandsen, S., \& Lindberg, B. 1999, Astrophysics with the NOT (Piikkio, Finland: Univ. Turku, Tuorla Observatory), 71

Fridlund, M., Gaidos, E., Barragán, O., et al. 2017, A\&A, 604, A16

Fuhrmann, K. 2004, AN, 325, 3

Fujii, Y., Del Genio, A. D., \& Amundsen, D. S. 2017, ApJ, 848, 100

Fukui, A., Livingston, J., Narita, N., et al. 2016, AJ, 152, 171

Fulton, B. J., Petigura, E. A., Howard, A. W., et al. 2017, AJ, 154, 109

Gandolfi, D., Alcalá, J. M., Leccia, S., et al. 2008, ApJ, 687, 1303

Gandolfi, D., Barragán, O., Hatzes, A. P., et al. 2017, AJ, 154, 123

Gandolfi, D., Parviainen, H., Deeg, H. J., et al. 2015, A\&A, 576, A11

Girardi, L., Groenewegen, M. A. T., Hatziminaoglou, E., \& da Costa, L. 2005 A\&A, 436, 895

Guenther, E. W., Barragán, O., Dai, F., et al. 2017, A\&A, 608, A93 
Henden, A. A., Templeton, M., Terrell, D., et al. 2016, yCat, 2336, 0

Hirano, T., Dai, F., Gandolfi, D., et al. 2017, arXiv:1710.03239

Hirano, T., Fukui, A., Mann, A. W., et al. 2016, ApJ, 820, 41

Hirano, T., Masuda, K., Sato, B., et al. 2015, ApJ, 799, 9

Howell, S. B., Everett, M. E., Sherry, W., Horch, E., \& Ciardi, D. R. 2011, AJ, 142,19

Howell, S. B., Sobeck, C., Haas, M., et al. 2014, PASP, 126, 398

Kasting, J. F., Whitmire, D. P., \& Reynolds, R. T. 1993, Icar, 101, 108

Kharchenko, N. V., Scholz, R.-D., Piskunov, A. E., Röser, S., \& Schilbach, E. 2007, AN, 328, 889

Kopparapu, R. k., Wolf, E. T., Arney, G., et al. 2017, ApJ, 845, 5

Kopparapu, R. K., Wolf, E. T., Haqq-Misra, J., et al. 2016, ApJ, 819, 84

Kovács, G., Zucker, S., \& Mazeh, T. 2002, A\&A, 391, 369

Lissauer, J. J., Marcy, G. W., Rowe, J. F., et al. 2012, ApJ, 750, 112

Livingston, J. H., Dai, F., Hirano, T., et al. 2017, arXiv:1710.07203

Luger, R., \& Barnes, R. 2015, AsBio, 15, 119

Mann, A. W., Feiden, G. A., Gaidos, E., Boyajian, T., \& von Braun, K. 2015 , ApJ, 804, 64

McQuillan, A., Mazeh, T., \& Aigrain, S. 2014, ApJS, 211, 24

Miller-Ricci, E., Seager, S., \& Sasselov, D. 2009, ApJ, 690, 1056

Montet, B. T., Morton, T. D., Foreman-Mackey, D., et al. 2015, ApJ, 809, 25

Moro, D., \& Munari, U. 2000, A\&AS, 147, 361

Morton, T. D. 2012, ApJ, 761, 6

Morton, T. D. 2015, VESPA: False Positive Probabilities Calculator, Astrophysics Source Code Library, ascl:1503.011

Niraula, P., Redfield, S., Dai, F., et al. 2017, AJ, 154, 266
Ohta, Y., Taruya, A., \& Suto, Y. 2009, ApJ, 690, 1

Perryman, M. A. C., Brown, A. G. A., Lebreton, Y., et al. 1998, A\&A, 331, 81

Press, W. H., Teukolsky, S. A., Vetterling, W. T., \& Flannery, B. P. 1992, Numerical Recipes in C. The Art of Scientific Computing (Cambridge: Cambridge Univ. Press)

Rogers, L. A. 2015, ApJ, 801, 41

Skrutskie, M. F., Cutri, R. M., Stiening, R., et al. 2006, AJ, 131, 1163

Telting, J. H., Avila, G., Buchhave, L., et al. 2014, AN, 335, 41

Tian, F., \& Ida, S. 2015, NatGe, 8, 177

Tody, D. 1986, Proc. SPIE, 627, 733

Tody, D. 1993, in ASP Conf. Ser. 52, Astronomical Data Analysis Software and Systems II, ed. R. J. Hanisch, R. J. V. Brissenden, \& J. Barnes (San Francisco, CA: ASP), 173

Tull, R. G., MacQueen, P. J., Sneden, C., \& Lambert, D. L. 1995, PASP, 107,251

Vanderburg, A., \& Johnson, J. A. 2014, PASP, 126, 948

Van Eylen, V., Agentoft, C., Lundkvist, M. S., et al. 2017, arXiv:1710.05398

Van Eylen, V., \& Albrecht, S. 2015, ApJ, 808, 126

Vida, K., Kővári, Z., Pál, A., Oláh, K., \& Kriskovics, L. 2017, ApJ, 841, 124

Way, M. J., Aleinov, I., Amundsen, D. S., et al. 2017, ApJS, 231, 12

Weiss, L. M., \& Marcy, G. W. 2014, ApJL, 783, L6

Wright, E. L., Eisenhardt, P. R. M., Mainzer, A. K., et al. 2010, AJ, 140, 1868

Yang, J., Cowan, N. B., \& Abbot, D. S. 2013, ApJL, 771, L45

Yee, S. W., Petigura, E. A., \& von Braun, K. 2017, ApJ, 836, 77

Zacharias, N., Finch, C., \& Frouard, J. 2017, AJ, 153, 166 